\title{
A novel acetyl xylan esterase enabling complete deacetylation of substituted xylans
}

\author{
Fakhria M. Razeq ${ }^{1}$, Edita Jurak², Peter J. Stogios ${ }^{1}$, Ruoyu Yan', Maija Tenkanen³ ${ }^{3}$ Mirjam A. Kabel ${ }^{4}$, Weijun Wang ${ }^{1}$ \\ and Emma R. Master ${ }^{1,2^{*}}$ (i)
}

\begin{abstract}
Background: Acetylated 4-O-(methyl)glucuronoxylan (GX) is the main hemicellulose in deciduous hardwood, and comprises a $\beta$ - $(1 \rightarrow 4)$-linked xylopyranosyl $(X y \mid p)$ backbone substituted by both acetyl groups and $a-(1 \rightarrow 2)$-linked 4-O-methylglucopyranosyluronic acid (MeGlcpA). Whereas enzymes that target singly acetylated Xylp or doubly 2,3-O-acetyl-Xylp have been well characterized, those targeting (2-O-MeGlcpA)3-O-acetyl-Xylp structures in glucuronoxylan have remained elusive.
\end{abstract}

Results: An unclassified carbohydrate esterase (FjoAcXE) was identified as a protein of unknown function from a polysaccharide utilization locus (PUL) otherwise comprising carbohydrate-active enzyme families known to target xylan. FjoAcXE was shown to efficiently release acetyl groups from internal (2-O-MeGlcpA)3-O-acetyl-Xylp structures, an activity that has been sought after but lacking in known carbohydrate esterases. FjoAcXE action boosted the activity of a-glucuronidases from families GH67 and GH115 by five and nine times, respectively. Moreover, FjoAcXE activity was not only restricted to GX, but also deacetylated (3-O-Araf)2-O-acetyl-Xylp of feruloylated xylooligomers, confirming the broad substrate range of this new carbohydrate esterase.

Conclusion: This study reports the discovery and characterization of the novel carbohydrate esterase, FjoAcXE. In addition to cleaving singly acetylated Xylp, and doubly 2,3-O-acetyl-Xylp, FjoAcXE efficiently cleaves internal 3-O-acetyl-Xylp linkages in (2-O-MeGlcpA)3-O-acetyl-Xylp residues along with densely substituted and branched xylooligomers; activities that until now were missing from the arsenal of enzymes required for xylan conversion.

Keywords: Acetyl xylan esterase, a-Glucuronidase, Glucuronic acid, Polysaccharide utilization loci, Xylan, SGNH hydrolase

\section{Background}

Hemicelluloses represent the second main polysaccharide component in lignocellulosic biomass after cellulose. In addition to a source of carbohydrates for fuels and chemicals, reported applications of hemicelluloses range from rheology modifiers and packaging films, to hydrogels as well as nutrient additives in food and feed $[1,2]$. Despite their broad application potential, hemicelluloses remain relatively underutilized, in part due to their diverse and heterogeneous compositions [3].

\footnotetext{
*Correspondence: emma.master@utoronto.ca

${ }^{1}$ Department of Chemical Engineering and Applied Chemistry, University of Toronto, 200 College Street, Toronto, ON M5S 3E5, Canada

Full list of author information is available at the end of the article
}

4-O-Methyl-glucuronoxylan (GX) is the main hemicellulose in deciduous trees, and contains $\beta$-( $(1 \rightarrow 4)$-linked xylopyranosyl $(\mathrm{Xyl} p)$ backbone units that can be acetylated and/or substituted by $\alpha$-(1 $\rightarrow 2)$-linked 4-O-methylglucopyranosyluronic acid (MeGlc $p$ A). Although species variations occur, approximately one of every ten Xylp units are substituted with MeGlc $p \mathrm{~A}$, and six of every ten $\mathrm{Xyl} p$ are acetylated at the $O-2$ and/or $O-3$ positions [47] (Fig. 1). By contrast, glucuronoarabinoxylans (GAX) dominate in cereal grains such as corn, and are characterized by $\mathrm{Xyl} p$ backbone residues that are decorated with L-arabinofuranosyl substituents at either the $\mathrm{O}-3$ or both $O-2$ and $O-3$ positions. To a lesser extent, these xylans can be substituted with 5-O-trans-feruloyl-L-arabinofuranose and/or an oligomeric side chain at the $\mathrm{O}-3$ 


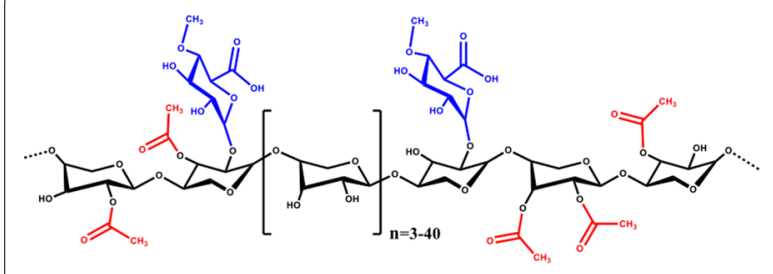

Hardwood: Glucuronoxylan (GX)

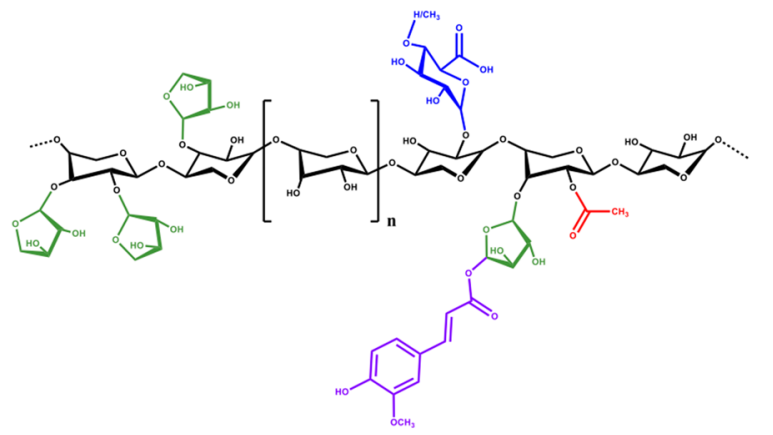

Grasses and cereals: Glucuronoarabinoxylan (GAX)

Fig. 1 Schematic structures of major xylan types. The xylosyl backbone is shown in black and ramifications with acetyl groups (red), 4-O-methylglucopyranosyluronic acid (MeGlcpA; blue) and arabinosyl units (green) linked with ferulic acid groups (purple) are indicated

position [8], as well as GlcpA and MeGlcpA residues and acetyl groups at the $O-2, O-3$ or both positions of $\mathrm{Xyl} p$ subunits [9-11] (Fig. 1). The presence of side groups along the xylan backbone impact several properties of corresponding biopolymers, including water solubility, rheology, adsorption behavior, nutrient value, and enzymatic conversion to monosaccharides [12].

Enzymatic conversion of xylans to monosaccharides, oligosaccharides, or defined polymeric structures requires the concerted action of carbohydrate-active enzymes (CAZymes; http://www.cazy.org) from multiple CAZyme families [13]. CAZymes that target side groups of GX and GAX include the following: $\alpha$-L-arabinofuranosidases from glycoside hydrolase $(\mathrm{GH})$ families $\mathrm{GH} 3, \mathrm{GH} 43$, GH51, GH54, and GH62, $\alpha$-glucuronidases from the families GH67 and GH115, and acetyl xylan esterases (AcXEs) that have been primary reported in carbohydrate esterase (CE) families CE1, CE4-CE6, and CE16 [14-18]. Whereas $\alpha$-glucuronidases from family GH115 target the $\alpha-(1 \rightarrow 2)$-linkage between MeGlc $p \mathrm{~A}$ and mono-substituted Xyl $p$ at internal and end positions of GX, GH67 activity is restricted to such linkages at the non-reducing end of xylan and/or corresponding oligosaccharides [7]. Similarly, CEs acting on GX have been grouped according to the positions they target (e.g., acting on $O-2$ and $O-3$ monoacetylated Xyl $p$, versus targeting 2,3-di-O-acetylated $\mathrm{Xyl} p$ ) [19]. Certain family CE16 enzymes also show activity towards non-reducing (2-O-MeGlcpA)3-O-acetyl-Xyl $p$ positions [19-22]; however, in these cases, acetyl group migration from $O-3$ to $O-4$ on the non-reducing $\mathrm{Xyl} p$ could not be ruled out $[5,19,23]$. Critically, to date, there are no known esterases that efficiently target internal (2-O-MeGlcpA)3-Oacetyl-Xyl $p$ structures, nor $\alpha$-glucuronidases that release MeGlcpA from acetylated Xyl $p$, which hinders selective release of MeGlcpA substituents for material applications and full conversion of GX to fermentable monosaccharides [7, 19, 24]. Similarly, CEs capable of targeting acetyl groups adjacent to arabinosyl substituents and oligomeric structures in GAX remain to be uncovered.

Polysaccharide utilization loci (PULs) comprise a physically linked set of bacterial genes that encode CAZymes and other proteins that work in concert to modify and degrade specific polysaccharides and/or oligosaccharides [25]. Predicted PULs were introduced to the CAZyme database in 2015 (http://www.cazy.org/PULDB/) [26], and have emerged as especially rich regions within genome and metagenome sequences for enzyme discovery. For example, within the last year alone, novel activities towards pectin [27], xylan [28], galactomannan [29], chitin [30], and $\beta$-glucans [31] were discovered.

Herein, we mined PUL sequences with the specific aim to finally uncover carbohydrate esterases that efficiently target (2-O-MeGlcpA)3-O-acetyl-Xyl $p$ positions internal to GX. Briefly, we identified PULs listed in the PULDB that encode CAZyme families known to target GX (i.e., GH10, GH115), and recombinantly expressed those sequences marked as having unknown function along with a predicted signal sequence for secretion. Ultimately, FjoAcXE encoded by Flavobacterium johnsoniae was selected and shown to cleave acetyl groups of internal and terminal (2-O-MeGlcpA)3-O-acetyl-Xyl $p$ structures of GX, leading to synergistic impacts on measured activities for both family GH67 and GH115 $\alpha$-glucuronidases. The discovery of FjoAcXE thus completes the arsenal of CAZymes required for GX conversion to defined structures or full hydrolysis to monosaccharides. Moreover, the deacetylation activity of FjoAcXE on xylooligomers from corn fiber confirmed its ability to target acetylated $\mathrm{Xyl} p$ structures also substituted by an oligomeric side chain, demonstrating the relevance of this unclassified $\mathrm{CE}$ activity for the conversion of diverse, complex xylans. 


\section{Methods}

\section{Materials}

para-Nitrophenol ( $p \mathrm{NP}$ ) alkyl esters were purchased from the following sources: $p$-nitrophenyl acetate $\left(C_{2}\right)$ (Sigma, St. Louis, MO, USA; N8130), $p$-nitrophenyl butyrate $\left(\mathrm{C}_{4}\right)$ (Sigma, N9876), $p$-nitrophenyl hexanoate $\left(\mathrm{C}_{6}\right)$ (TCI-EP, Tokyo, Japan; H0484), p-nitrophenyl octanoate $\left(\mathrm{C}_{8}\right)$ (Sigma, 21742), p-nitrophenyl decanoate $\left(\mathrm{C}_{10}\right)$ (Sigma, N0252), p-nitrophenyl dodecanoate $\left(\mathrm{C}_{12}\right)$ (Sigma, 61716), $p$-nitrophenyl myristate $\left(\mathrm{C}_{14}\right)$ (Sigma, 70124), and $p$-nitrophenyl palmitate $\left(\mathrm{C}_{16}\right)$ (Sigma, N2752). In addition, 4-methylumbelliferyl acetate (4-MUA) was purchased from Sigma (M0883) and 4-methylumbelliferone (4-MU) was purchased from Aldrich (M1381).

Acetylated monosaccharide substrates were purchased from the following sources: $\beta$-D-glucose pentaacetate (Aldrich, 285943), $\beta$-D-xylopyranose tetraacetate (Synthose Inc., Toronto, ON, Canada; TX534) and 1,2,3,4-tetra-O-acetyl-L-rhamnopyranose (Synthose Inc., TH212). Acetylated glucurono-xylooligosaccharides (Ac-XOS) were isolated by steam extraction of milled chips from Eucalyptus wood [32], or mixed hardwood kindly provided by Prof. Bradley Saville (University of Toronto, Canada) [33]. Isolation and characterization of xylooligosaccharides from corn fibers were previously described [8].

The family GH115 $\alpha$-glucuronidase from Amphibacillus xylanus (AxyAgu115) was purified as previously described [34]. The family CE6 acetylxylan esterase from Orpinomyces sp. (E-AXEAO) was purchased from Megazyme (Bray, Ireland); the family GH67 $\alpha$-glucuronidase from Cellvibrio japonicus (PRO-E0069; CjGlcA67A) was purchased from PROZOMIX Ltd (Haltwhistle, UK).

\section{Candidate selection}

The PUL database (PULDB, http://www.cazy.org/PULDB) [26] was searched for PULs that contained at least two of the predicted xylan-active CAZyme families of interest, such as GH10, GH43, GH67, and GH115. From the obtained list of PULs, all proteins of unknown function were extracted and analyzed using Signal P4.1 [35]; those containing a predicted signal sequence for secretion were further analyzed based on the presence of Pfam domains, sequence length, and the type of CAZymes present on the corresponding PUL. Based on these criteria, FjoAcXE (PULDB ID: Fjoh_3879; GenBank ID: ABQ06890.1) was selected for recombinant protein expression. A structural model of FjoAcXE was built using PHYRE2.0 [36] for the CBM-like domain, using PDB 2O14 as template, and Modeller 9.19 [37] for the CE/ catalytic domain using PDB $2 \mathrm{O} 14$ and PDB 1 K7C [38]. The model was displayed with PyMOLv1.7.4.5 Edu (PyMOL Molecular Graphics System, Schrödinger, LLC).

\section{Gene synthesis and molecular cloning}

The gene encoding FjoAcXE lacking the predicted signal sequence (residues 1-21) was codon optimized for expression in Escherichia coli K12 using IDT Codon Optimization Tool (http://www.idtdna.com/CodonOpt). Fifteen base pair extensions homologous to the p15TVL vector (GenBank ID: EF456736.1) (T7, 5'-TTGTAT TTCCAGGGC and T7term, 5'-CAAGCTTCGTCA TCA) were added to each end of the sequence and the gene was synthesized as gBlock ${ }^{\circledR}$ gene fragments (Integrated DNA Technologies, Inc., Coralville, IA, USA). gBlock fragments were cloned into p15TV-L using the In-Fusion ${ }^{\circledR}$ HD EcoDry ${ }^{\text {TM }}$ Cloning Kit (Clontech Laboratories, Inc., Palo Alto, CA, USA). The resulting plasmid was transformed into E. coli HST08 Stellar ${ }^{\mathrm{TM}}$ Competent Cells (Clontech Laboratories, Inc.), and the sequence was verified using DNA sequencing service at the Center of Applied Genomics at the SickKids Hospital (Toronto, ON, Canada).

\section{Protein expression and purification}

E. coli BL21( $\lambda \mathrm{DE} 3)$ codon plus strain harboring p15TVL-FjoAcXE was propagated at $37^{\circ} \mathrm{C}$ in $8 \mathrm{~L}$ of Luria-Bertani (LB) Broth-Miller (BioShop) supplemented with $33 \mu \mathrm{g} / \mathrm{mL}$ chloramphenicol and $100 \mu \mathrm{g} / \mathrm{mL}$ ampicillin until the $\mathrm{OD}_{600}$ reached $0.6-0.8$. Cultures were cooled on ice for $5 \mathrm{~min}$; additional $33 \mu \mathrm{g} / \mathrm{mL}$ chloramphenicol and $100 \mu \mathrm{g} / \mathrm{mL}$ ampicillin were added, and recombinant expression was induced overnight at $16{ }^{\circ} \mathrm{C}$ with $0.5 \mathrm{mM}$ isopropyl $\beta$-D-1-thiogalactopyranoside. Cells were harvested by centrifugation at $8967 \times g$ (Beckman Coulter, JLA-8.1000) for $15 \mathrm{~min}$ at $4{ }^{\circ} \mathrm{C}$ and the pellet (approx. $25 \mathrm{~g}$ fresh weight) was frozen at $-80{ }^{\circ} \mathrm{C}$. The pellet was then suspended in binding buffer (50 mM HEPES pH 7.5, $300 \mathrm{mM} \mathrm{NaCl}, 5 \%$ glycerol and $5 \mathrm{mM}$ imidazole) and the cells were disrupted by sonication (100 amplitude, $5 \mathrm{~s}$ ON and $5 \mathrm{~s}$ OFF for $20 \mathrm{~min}$ ). Cell debris was removed by centrifugation at $27,167 \times g$ (Beckman Coulter, JA-25.5 rotor) for $15 \mathrm{~min}$ at $4{ }^{\circ} \mathrm{C}$ and supernatant was filtered through Acrodisc ${ }^{\circledR}$ Syringe Filters with $0.45-\mu \mathrm{m}$ Supor ${ }^{\circledR}$ membrane (Pall Corporation).

The sample was loaded onto 5-mL HisTrap HP (GE Healthcare) pre-equilibrated with binding buffer. A combination of step-wise and gradient elutions using elution buffer (50 mM HEPES pH 7.5, $300 \mathrm{mM} \mathrm{NaCl}, 5 \%$ glycerol and $300 \mathrm{mM}$ imidazole) was performed on Biologic DuoFlow $^{\text {TM }}$ chromatography system (BioRad) where the column was washed with 4 column volumes (CV) of $100 \%$ binding buffer, and then increasingly substituted with elution buffer as follows: $5 \%$ elution buffer for $4 \mathrm{CV}, 10 \%$ elution buffer for $6 \mathrm{CV}$ and then gradient elution from 10 to $100 \%$ elution buffer over $12 \mathrm{CV}$. All steps were done at $1 \mathrm{~mL} / \mathrm{min}$, protein elution was monitored at $A_{280 \mathrm{~nm}}$ and 
$2 \mathrm{~mL}$ fractions were collected throughout the entire run. The resulting fractions were analyzed by $12 \%$ SDS-PAGE; selected fractions were pooled and then exchanged to 25 mM HEPES (pH 8.0) using 10 kDA Jumbosep ${ }^{\mathrm{TM}}$ centrifugal devices (Pall Corporation). The sample was then further purified using a 1.3-mL $\mathrm{UNO}^{\mathrm{TM}} \mathrm{Q}$ ion exchange column (BioRad) pre-equilibrated with 25 mM HEPES pH 8.0 (Buffer A). Following a wash using $11.5 \mathrm{CV}$ of Buffer A, a step-wise gradient elution was performed at $1 \mathrm{~mL} / \mathrm{min}$ on a Biologic DuoFlow ${ }^{\mathrm{TM}}$ using $25 \mathrm{mM}$ HEPES pH 8.0 with $1 \mathrm{M} \mathrm{NaCl}$ as elution buffer (Buffer B), where $0-50 \%$ of Buffer B was passed over 15.4 CV, and then up to $100 \%$ Buffer B over $6 \mathrm{CV}$. The samples were collected as $1-\mathrm{mL}$ fractions throughout the entire run and purity was checked with $12 \%$ SDS-PAGE. The purified sample was exchanged to $10 \mathrm{mM}$ HEPES $\mathrm{pH} 7.5$ containing $300 \mathrm{mM} \mathrm{NaCl}$ before being flash frozen in liquid nitrogen and stored at $-80^{\circ} \mathrm{C}$.

Protein concentration was measured using the Bradford assay with bovine serum albumin as a standard [39]. The identity of purified FjoAcXE was then confirmed by peptide mass fingerprinting using an easy-nLC-1000 (ThermoFisher Scientific, Bremen, Germany) equipped with a 10.5-cm PicoTip Emitter Silica Tip packed inhouse with $\mathrm{C} 18$ media coupled online to a Q-Exactive mass spectrometer (ThermoFisher Scientific) [40].

\section{Enzyme activity measurements using $p N P$ aliphatic fatty acid esters and 4-MUA}

$200 \mathrm{mM}$ stock solutions for $p \mathrm{NP}$ alkyl esters from $\mathrm{C} 2$ to $\mathrm{C} 12$ were prepared in $100 \%$ DMSO, whereas $p$ NP substrates from C14 to C16 were prepared in 1:1 (v/v) of isopropanol:acetonitrile. Reactions were performed in $50 \mathrm{mM}$ HEPES ( $\mathrm{pH} 7.0$ ) with $2 \mathrm{mM}$ substrate and were initiated by adding $0.5 \mu \mathrm{g}$ of FjoAcXE; the final reaction volume was $200 \mu \mathrm{L}$. Reactions were conducted at $\mathrm{pH} 7.0$ to minimize non-enzymatic de-esterification of $p$ NP substrates over the prolonged incubation (i.e., $2 \mathrm{~h}$ ). Reactions continued for $2 \mathrm{~h}$ at $30{ }^{\circ} \mathrm{C}$, and absorbance was measured continuously at $410 \mathrm{~nm}$. Reaction mixtures without FjoAcXE were used as a blank.

Kinetic parameters of FjoAcXE were determined using $p \mathrm{NP}$-acetate and 4-MUA. For $p \mathrm{NP}$-acetate, reactions contained approximately $0.06 \mu \mathrm{g}\left(1.32 \times 10^{-6} \mu \mathrm{mol}\right)$ of FjoAcXE in $50 \mathrm{mM}$ HEPES (pH 8.0) and were initiated by adding $0.05 \mathrm{mM}$ to $10 \mathrm{mM} p \mathrm{NP}$-acetate. The final reaction volume was $200 \mu \mathrm{L}$, and absorbance at $410 \mathrm{~nm}$ was read continuously for $40 \mathrm{~min}$ at $30^{\circ} \mathrm{C}$.

For 4-MUA, reactions contained approximately $0.02 \mu \mathrm{g}$ $\left(4.41 \times 10^{-7} \mu \mathrm{mol}\right)$ of FjoAcXE in $50 \mathrm{mM}$ HEPES ( $\left.\mathrm{pH} 8.0\right)$ and final of $10 \%$ DMSO. The enzyme dose was chosen to ensure a linear relationship between reaction time and product release [41]; reactions were then initiated by adding $0.01-1.5 \mathrm{mM}$ of 4-MUA. The final reaction volume was $200 \mu \mathrm{L}$, and absorbance at $354 \mathrm{~nm}$ was read continuously for $40 \mathrm{~min}$ at $30{ }^{\circ} \mathrm{C}$. Kinetic parameters were calculated using Michaelis-Menten equation and Graphpad Prism 5 software (La Jolla, CA, USA).

\section{Optimum reaction conditions}

The $\mathrm{pH}$ optimum of FjoAcXE was tested using $100 \mathrm{mM}$ Tris, $50 \mathrm{mM}$ MES, $50 \mathrm{mM}$ acetic acid, and $50 \mathrm{mM}$ sodium acetate trihydrate buffer with $\mathrm{pH}$ range of 3.59.5. Reactions containing $0.5 \mathrm{mM}$ 4-MUA were initiated by adding $5 \mu \mathrm{g}$ of FjoAcXE, and continued for $20 \mathrm{~min}$ at $30{ }^{\circ} \mathrm{C}$; the final reaction volume was $200 \mu \mathrm{L}$. 4-MUA was selected to determine the $\mathrm{pH}$ optimum of FjoAcXE given the relative stability of this substrate under alkaline conditions [41]. For all reactions using 4-MUA, absorbance was measured at $354 \mathrm{~nm}$ and the reaction mixture without FjoAcXE was used as a blank.

To measure $\mathrm{pH}$ stability, solutions containing $4 \mu \mathrm{g}$ of FjoAcXE were incubated for $16 \mathrm{~h}$ at $4{ }^{\circ} \mathrm{C}$ in $100 \mathrm{mM}$ Tris, $50 \mathrm{mM}$ MES, $50 \mathrm{mM}$ acetic acid, and $50 \mathrm{mM}$ sodium acetate trihydrate buffer adjusted to $\mathrm{pH}$ 3.5-9.5; the final solution volume was $40 \mu \mathrm{L}$. After incubation, residual enzyme activity was tested in reactions containing $0.5 \mathrm{mM}$ 4-MUAc in $50 \mathrm{mM}$ HEPES (pH 8.0). Reactions were initiated by adding $1 \mu \mathrm{g}$ of FjoAcXE from each treatment and continued for $20 \mathrm{~min}$ at $30^{\circ} \mathrm{C}$; the final reaction volume was $200 \mu \mathrm{L}$.

The temperature stability of FjoAcXE was tested by suspending $4 \mu \mathrm{g}$ of FjoAcXE in 50 mM HEPES ( $\mathrm{pH}$ 8.0) and incubating each suspension ( $40 \mu \mathrm{L}$ final volume) for up to $16 \mathrm{~h}$ at 20,30 , or $40^{\circ} \mathrm{C}$, or up to $60 \mathrm{~min}$ at 50,60 , or $70{ }^{\circ} \mathrm{C}$. After the incubation, residual activity was tested in reactions containing $0.5 \mathrm{mM} 4-\mathrm{MUAc}$ in $50 \mathrm{mM}$ HEPES (pH 8.0). Similar to $\mathrm{pH}$ stability, reactions were initiated by adding $1 \mu \mathrm{g}$ of FjoAcXE from each treatment, and continued for $20 \mathrm{~min}$ at $30^{\circ} \mathrm{C}$; the final reaction volume was $200 \mu \mathrm{L}$.

\section{Effect of divalent ions, detergents, and organic solvents}

The metal-free apoenzyme of FjoAcXE was prepared as described in Wang et al. [42]. Briefly, $0.05 \mathrm{~g}$ of CHELEX 100 (Sigma) was added to $1 \mathrm{mg}$ of FjoAcXE and incubated at room temperature for $20 \mathrm{~min}$. The effect of metal ions was tested in reactions containing $0.5 \mathrm{mM}$ 4-MUA in $50 \mathrm{mM}$ HEPES (pH 8.0). Reactions were initiated by adding $5 \mu \mathrm{g}$ of FjoAcXE, and continued for $20 \mathrm{~min}$ at $30^{\circ} \mathrm{C}$ in the presence of $1 \mathrm{mM}$ of the following metal ions: $\mathrm{Ag}^{2+}$, $\mathrm{Ca}^{2+}, \mathrm{Cd}^{2+}, \mathrm{Co}^{2+}, \mathrm{Cu}^{2+}, \mathrm{Fe}^{3+}, \mathrm{Mg}^{2+}, \mathrm{Mn}^{2+}, \mathrm{Ni}^{2+}$, and $\mathrm{Zn}^{2+}$ (all as chloride salts, except for $\mathrm{Ag}^{2+}$, which was a nitrate). The final reaction volume was $200 \mu \mathrm{L}$, and control samples included CHELEX treated and untreated FjoAcXE with and without $20 \mathrm{mM}$ EDTA. Absorbance 
was measured at $354 \mathrm{~nm}$ and the reaction mixture without FjoAcXE was used as a blank.

The chemical stability of FjoAcXE was tested in reactions containing $0.5 \mathrm{mM}$ 4-MUAc in $50 \mathrm{mM}$ HEPES $(\mathrm{pH}$ 8.0). Reactions were initiated by adding $0.5 \mu \mathrm{g}$ of FjoAcXE, and continued for $20 \mathrm{~min}$ at $30{ }^{\circ} \mathrm{C}$ in the presence of $30 \%(\mathrm{v} / \mathrm{v})$ ethanol, 30\% (v/v) isopropanol, $1 \%(\mathrm{w} / \mathrm{v})$ SDS, $1 \%(\mathrm{w} / \mathrm{v})$ dithiothreitol (DTT), 1\% (v/v) Tween-20, 1\% (v/v) Tween-80, and 1\% (v/v) TritonX-100; the final reaction volume was $200 \mu \mathrm{L}$.

\section{Activity on acetylated monosaccharides}

Reactions comprised $50 \mathrm{mM}$ HEPES (pH 8.0) and 0.1\% $(\mathrm{w} / \mathrm{v})$ of $\beta$-D-glucose pentaacetate (final concentration $2.5 \mathrm{mM}$ ), $\beta$-D-xylopyranose tetraacetate (final concentration $3.14 \mathrm{mM}$ ), or 1,2,3,4-tetra-O-acetyl-L-rhamnopyranose (final concentration $3 \mathrm{mM}$ ). Reactions were initiated by adding $0.5 \mu \mathrm{g}$ of FjoAcXE; the final reaction volume was $30 \mu \mathrm{L}$. Reactions continued for $20 \mathrm{~min}$ at $30^{\circ} \mathrm{C}$, and were stopped by boiling for $10 \mathrm{~min}$. The samples were quickly centrifuged and release of acetic acid was then measured in the supernatant using the Acetic Acid kit (K-ACETRM, Megazyme). Reaction mixtures without FjoAcXE were used as a blank for each substrate.

\section{${ }^{1} \mathrm{H}$-NMR analysis of FjoAcXE activity on acetylated (glucurono)-xylooligosaccharides}

${ }^{1} \mathrm{H}$-NMR was performed to monitor the activity of FjoAcXE and AxyAgu115A on Ac-XOS substrates. Reactions comprised $1 \%(\mathrm{w} / \mathrm{v}$ ) acetylated (glucurono)-xylooligosaccharides in $50 \mathrm{mM}$ HEPES buffer (pH 7.0) and $10 \mu \mathrm{g}$ of each protein; the final reaction volume was $400 \mu \mathrm{L}$. The reaction continued for $20 \mathrm{~h}$ at $30{ }^{\circ} \mathrm{C}$ and gentle shaking. Reaction mixtures without enzyme were used as negative controls. Following the incubation, samples were filtered through Acrodisc ${ }^{\circledR}$ syringe filters with $0.2-\mu \mathrm{m}$ Supor ${ }^{\circledR}$ membrane (Pall Corporation), and lyophilized. The samples were then dissolved in $300 \mu \mathrm{L} \mathrm{D}_{2} \mathrm{O}$ and transferred into 3-mm NMR tubes (Norell) for analysis using an Agilent DD2 $700 \mathrm{MHz}$ spectrometer equipped with a triple resonance $\mathrm{HCN}$ cold probe with a scan number of 64 , relaxation delay of $1 \mathrm{~s}$ and acquisition time of $4.5 \mathrm{~s}$. The data were obtained using VnmrJ 4.0 (Agilent) and analyzed with MestReNova 10.0 (Mestrelab Research). The HDO peak at 4.790 was used as internal standard. The change in signal intensity in the regions between 5.4 and $4.4 \mathrm{ppm}$ corresponding to acetylated Xyl $p$ residues in the anomeric region of the spectrum, and 2.30-2.05 ppm region corresponding to the acetyl group methyl protons were used to assign proton chemical shifts, as reported in Uhliariková et al. [24].
Impact of FjoAcXE on a-glucuronidase activity towards (glucurono)-xylooligosaccharides

FjoAcXE, CjGlcA67A, AxyAgu115A, and E-AXEAO were tested alone and as pairs of carbohydrate esterase and $\alpha$-glucuronidase activities. Reactions comprised $1 \%$ (w/v) Ac-XOS in $50 \mathrm{mM}$ HEPES (pH 7.0), which is within one $\mathrm{pH}$ unit from the optimum $\mathrm{pH}$ of each enzyme. Reactions were initiated by adding $0.5 \mu \mathrm{g}$ of each enzyme; the final reaction volume was $30 \mu \mathrm{L}$. Reactions continued for $20 \mathrm{~min}$ at $30{ }^{\circ} \mathrm{C}$ and were stopped by boiling for $10 \mathrm{~min}$. The samples were quickly centrifuged and release of acetic acid and D-glucuronic acid were measured using Acetic Acid (K-ACETRM, Megazyme) and D-glucuronic acid/D-galacturonic acid (K-URONIC, Megazyme) assay kits, respectively. Reaction mixtures without enzymes were used as blanks.

\section{Matrix assisted laser desorption time of flight mass spectrometry (MALDI-TOF) analysis of FjoAcXE activity on feruloylated xylooligosaccharides}

Reactions comprised 50 mM HEPES (pH 8.0), 1\% (w/v) of feruloylated xylooligomers from corn fiber, and were initiated by adding $1 \mu \mathrm{g}$ of FjoAcXE; the final reaction volume was $50 \mu \mathrm{L}$. Reactions continued for $20 \mathrm{~min}$ at $30{ }^{\circ} \mathrm{C}$ with gentle shaking, and were stopped by boiling the samples at $100{ }^{\circ} \mathrm{C}$ for $10 \mathrm{~min}$. Reaction mixtures without enzyme were used as negative controls. One microliter of each reaction sample (after desalting with AG 1-X8 anion exchange resin; Bio-Rad, Hercules, CA, USA) was then mixed with $2 \mu \mathrm{L}$ matrix solution [2,5-dihydroxybenzoic acid, $10 \mathrm{mg} / \mathrm{mL} \mathrm{H}_{2} \mathrm{O} / \mathrm{ACN}$ (3:7)] on a stainless-steel metal plate and allowed to dry under a constant stream of air. MALDI-TOF was performed using an Ultra-flex3 instrument (Bruker Daltonics, Bremen, Germany) equipped with a nitrogen laser of $337 \mathrm{~nm}$ and operated in the positive mode. The system was controlled by FlexAnalysis software. Calibration was performed with a mixture of maltodextrins 480-3000 Da (Elicityl, Crolles, France). After a delayed extraction time of $130 \mathrm{~ns}$, positive ions were accelerated with $22 \mathrm{kV}$ voltage and detected using reflector mode.

\section{Results and discussion}

Candidate selection and recombinant protein production

FjoAcXE was marked as a protein of unknown function within PUL20 from Flavobacterium johnsoniae UW 101 on PULDB, also comprising CAZyme families GH43, GH115, CE1 and CE6 (Fig. 2). In addition to a predicted signal sequence for secretion (residues 1-21), the FjoAcXE sequence contained a predicted SGNH hydrolase-type esterase domain belonging to GDSL-like lipase/ 


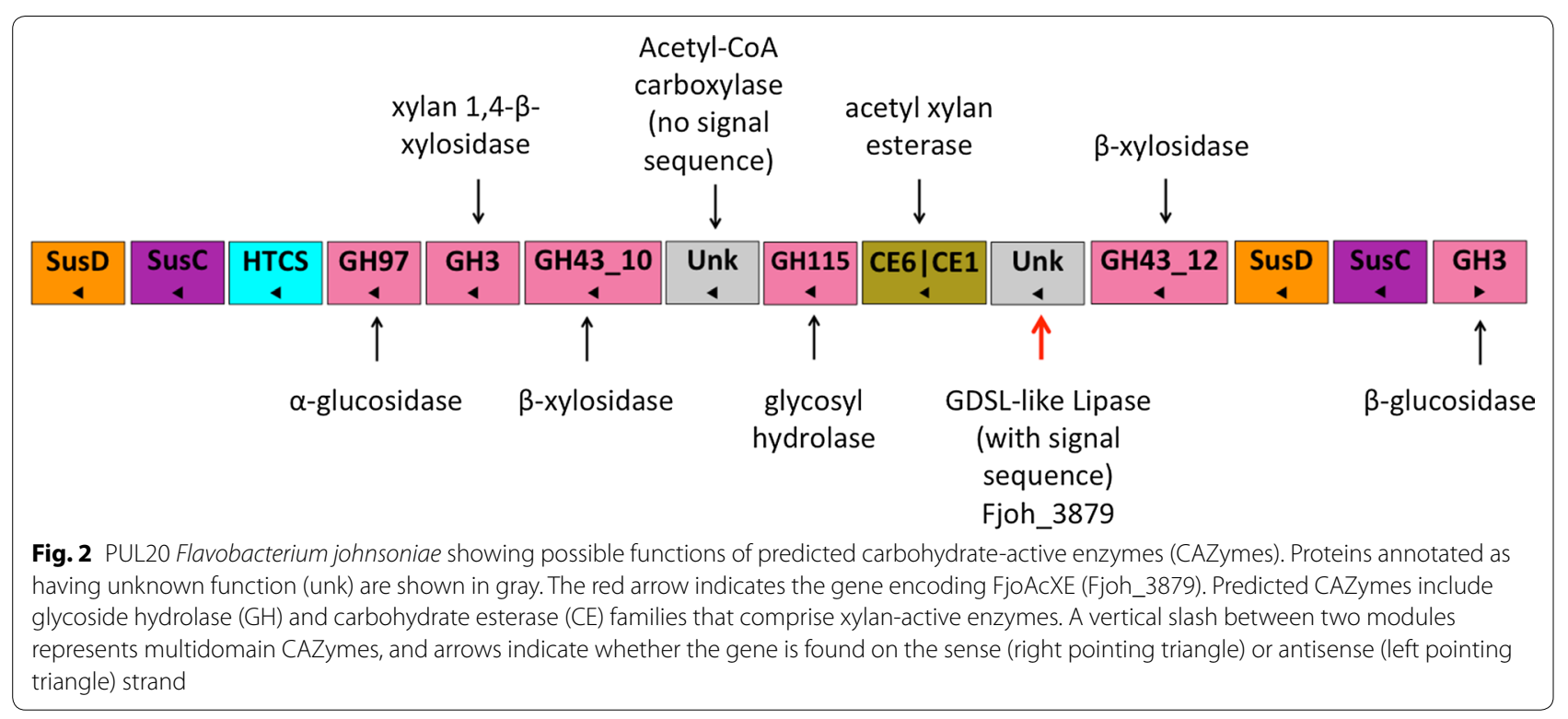

acylhydrolase family (Pfam domain PF13472) (Fig. 3). FjoAcXE shares less than $30 \%$ sequence identity to $\mathrm{CE}$ families listed in the CAZyme database and has not been assigned to a CAZyme family. Using Phyre2.0, however, FjoAcXE was predicted to adopt a structure most similar to CEs from families CE2 and CE12 (between residues 188-393, the C-terminus) as well as a CBM-like structure at the N-terminus (residues 59-180). Guided by the Phyre2.0 result, we generated a family of models of the FjoAcXE catalytic domain using CE12 enzyme structures as templates and the Modeller program; one such model is shown in Additional file 1: Fig. S1. The models recapitulated the $\alpha / \beta / \alpha$-fold characteristic of SGNH hydrolases and the position of the Ser-His-Asp triad; however, they varied in the conformation of three loops surrounding the catalytic site: residues 201-208, 238-241, and 368373. These loops are expected to shape the size of the catalytic pocket and play a role in substrate preference. In particular, the 238-241 region is located between the catalytic Ser196 and Asn273; the latter residue is thought to participate with Gly237 and the amide of Ser196 in forming an oxyanion hole to stabilize the $O$-acetylserine intermediate in the catalytic mechanism of CE enzymes [43]. As well, the 201-208 loop is located near the CBM-like domain and may play a role in substrate positioning in cooperation with that domain. The model places the catalytic site to face the CBM-like domain, indicating that this $\mathrm{N}$-terminal region could play a role in placement of substrates appropriately for catalysis (Additional file 1: Fig. S1). Notably, however, affinity gel electrophoresis (AGE) using a wide range of polysaccharides did not reveal carbohydrate binding by FjoAcXE (Additional file 2: Fig. S2). Nevertheless, the SGNH family displays a wide range of hydrolytic functions [44], and given our search for CE

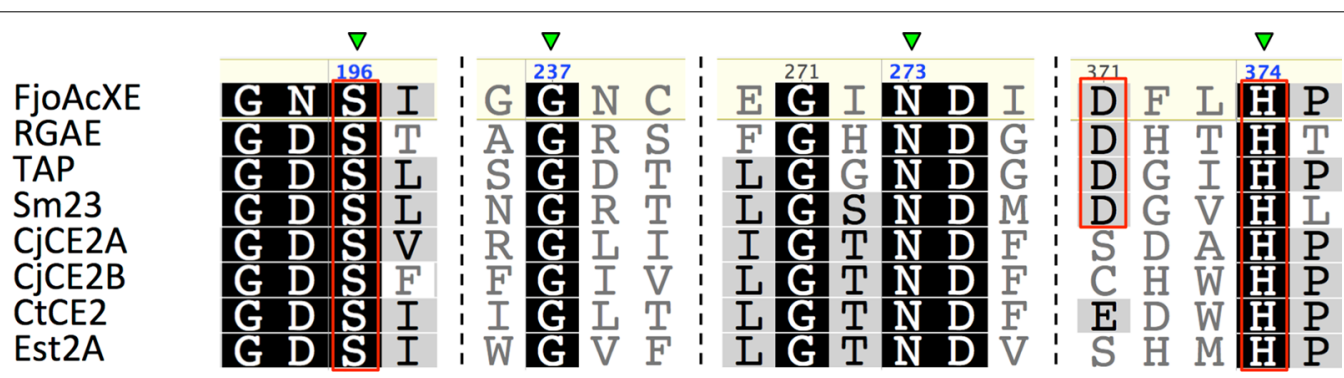

Fig. 3 Alignment of FjoACXE with structurally and biochemically characterized SGNH hydrolase-type esterases. RGAE: rhamnogalacturonan acetylesterase from Aspergillus aculeatus (PDB code 1DEO) [52]; TAP: Thioesterase I/Protease I/Lysophospholipase L1 from E. coli (PDB code 1IVN) [53, 54]; Sm23: arylesterase from Sinorhizobium meliloti (PDB code 4TX1) [55]; CjCE2A and CjCE2B: acetylxylan esterase from Cellvibrio japonicus (PDB codes 2WAA and2W9X, respectively) [43]; CtCE2: acetylxylan esterase from Ruminiclostridium thermocellum (PDB code 2WAO) [43]; Est2A: acetylxylan esterase from Butyrivibrio proteoclasticus (PDB code 3U37) [56]. Conserved SGNH residues (green triangle) and catalytic Ser-His dyad or Ser-Asp-His triad (red squares) are highlighted. Sequences were aligned using MAFFT v.7.017 [57] 
activities targeting resistant (2-O-MeGlcpA)3-O-acetyl$\mathrm{Xyl} p$ structures in GX, FjoAcXE was selected for recombinant production and biochemical characterization.

\section{General properties of FjoAcXE}

Recombinant FjoAcXE was functionally expressed in $E$. coli with an $\mathrm{N}$-terminal $\mathrm{His}_{6}$-tag and purified to homogeneity with approximate yield of $6 \mathrm{mg} / \mathrm{g}$ of fresh cell pellet. The predicted molecular mass of recombinant FjoAcXE is $45.2 \mathrm{kDa}$, which was consistent with that estimated by SDS-PAGE (Additional file 3: Fig. S3). Whereas no or negligible activity was measured on tested polysaccharides and $p$ NP-glycosides (Additional file 4: Fig. S4, Additional file 5: Fig. S5, respectively), clear activity was observed on $p \mathrm{NP}$-acetate followed by $p$ NP-butyrate
(Additional file 6: Fig. S6); notably, lack of activity on longer chain alkyl esters is consistent with esterase rather than lipase activity [45].

Given the similar activity of FjoAcXE on $p \mathrm{NP}$-acetate and 4-MUA (Table 1), and the relative stability of 4-MUA compared to $p \mathrm{NP}$-acetate [41], 4-MUA was used to evaluate the effect of $\mathrm{pH}$, temperature, metal ions, and chemical reagents on activity of FjoAcXE.

Whereas FjoAcXE activity was highest at $\mathrm{pH} 8.0$ (Fig. 4a), FjoAcXE retained over $80 \%$ of its activity after $16 \mathrm{~h}$ of pre-incubation at $\mathrm{pH} 4.5$ to $\mathrm{pH} 9.5$ (Fig. 4b). Furthermore, FjoAcXE retained $100 \%$ of its initial activity after $16 \mathrm{~h}$ at $20-40{ }^{\circ} \mathrm{C}$; only $50 \%$ activity was retained after $10 \mathrm{~min}$ at $50{ }^{\circ} \mathrm{C}$ and no residual activity was detected after $5 \mathrm{~min}$ at $60{ }^{\circ} \mathrm{C}$ (Fig. 4c). The biochemical

Table 1 Kinetic parameters of FjoAcXE on 4-MUA and pNP-acetate

\begin{tabular}{llccc}
\hline Substrate & Specific activity $(\boldsymbol{\mu m o l} / \mathbf{m i n} / \mathbf{m g})$ & $\boldsymbol{k}_{\text {cat }}\left(\mathbf{s}^{-\mathbf{1}}\right)$ & $\boldsymbol{K}_{\mathbf{m}}(\mathbf{m M})$ & $\boldsymbol{k}_{\text {cat }} / \boldsymbol{K}_{\mathbf{m}}\left(\mathbf{s}^{\mathbf{- 1}} \mathbf{m M} \mathbf{M}^{\mathbf{- 1}}\right)$ \\
\hline 4-MUA & $126.3 \pm 5.9$ & $95.1 \pm 4.4$ & $0.8 \pm 0.1$ & 114.7 \\
pNP-acetate & $160.7 \pm 4.0$ & $120.8 \pm 3.0$ & $0.9 \pm 0.1$ & 127.9 \\
\hline
\end{tabular}

$n=3$; errors represent standard deviation
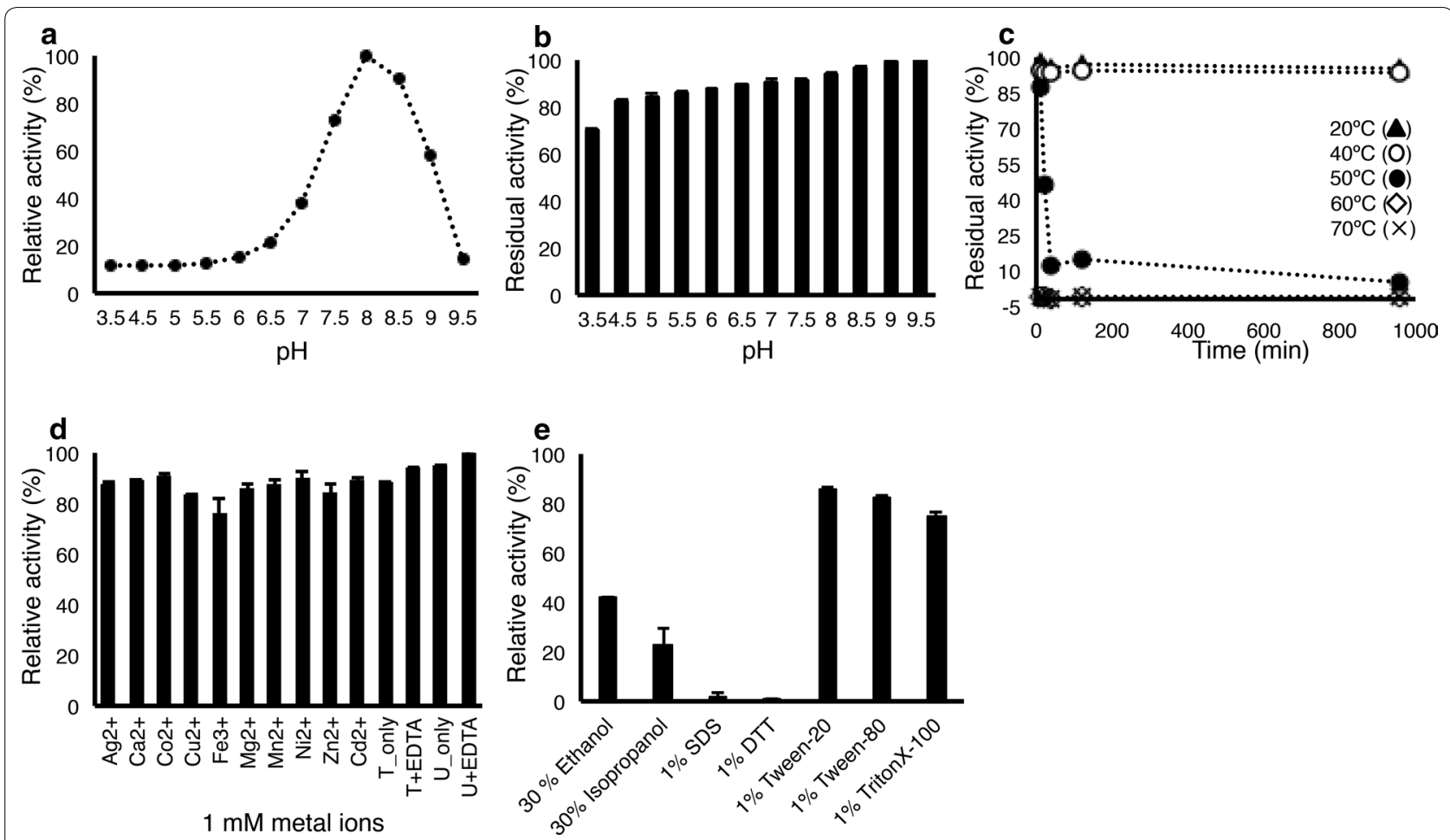

Fig. 4 General biochemical properties of FjoAcXE. For optimum pH (a) and pH stability (b), the samples were tested using $100 \mathrm{mM}$ Tris, $50 \mathrm{mM}$ MES, $50 \mathrm{mM}$ acetic acid, and $50 \mathrm{mM}$ sodium acetate trihydrate universal buffer, with pH range of 3.5-9.5. Temperature stability (c), effect of metal ions (d), and effect of selected solvents and reactants (e), were measured using 0.5 mM 4-MUA in 50 mM HEPES (pH 8.0) where reactions proceeded for $20 \mathrm{~min}$ at $30^{\circ} \mathrm{C}$. Absorbance at $354 \mathrm{~nm}$ was measured and the reaction mixture without protein was used as a blank. $n=3$; error bars correspond to standard deviation 


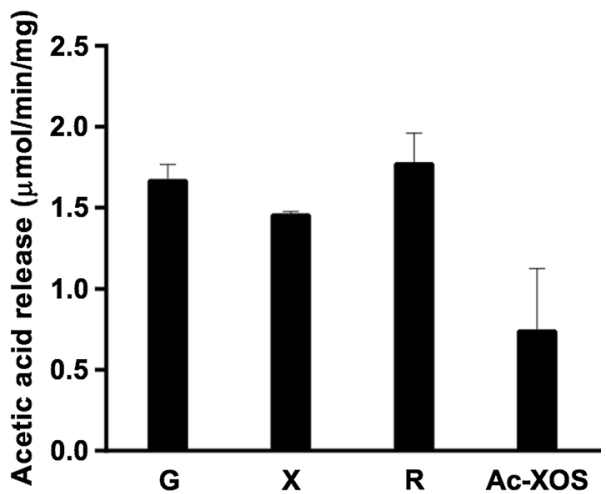

Fig. 5 Activity of FjoAcXE on acetylated monosaccharides and xylooligosaccharides. Reactions ( $30 \mu \mathrm{L}$ ) comprised $0.5 \mu \mathrm{g}$ of FjoAcXE in $50 \mathrm{mM} \mathrm{HEPES}(\mathrm{pH} 8.0)$ and $0.1 \%(\mathrm{w} / \mathrm{v})$ of $\beta$-D-glucose pentaacetate (G), $\beta$-D-xylopyranose tetraacetate (X), 1,2,3,4-tetra-O-acetyl-L-rhamnopyranose (R), or acetylated xylooligosaccharides from Eucalyptus (Ac-XOS); reactions were incubated at $30^{\circ} \mathrm{C}$ for $20 \mathrm{~min}$. Release of acetic acid was measured using acetic acid kit (K-ACETRM; Megazyme). $n=3$; error bars correspond to standard deviation properties of FjoAcXE were similar to previously reported SGHN hydrolases [46-49] and consistent with the soil and freshwater occurrence of the source organism (Flavobacterium johnsoniae). Most notably, FjoAcXE shows significant $\mathrm{pH}$ stability (Fig. 4b) compared to other reported SGNH hydrolases, such as Est19 from Bacillus sp., which shows substantial loss of activity after 1-h preincubation at $\mathrm{pH}$ below 6.0 and above 10.0 [50]. None of the metal ions tested significantly affected FjoAcXE activity (Fig. 4d) and addition of 1\% Tween-20, Tween-80, and Triton-X-100 resulted in only $30 \%$ loss of activity of FjoAcXE (Fig. 4e). The overall pH and surfactant stability of FjoAcXE may offer advantages in applications ranging from prebiotics, detergents, as well as biofuels [51].

\section{Activity of FjoAcXE on acetylated mono- and oligosaccharides}

Rates of FjoAcXE action towards fully acetylated monosaccharides and partially acetylated xylooligosaccharides were also comparable (Fig. 5), indicating that FjoAcXE

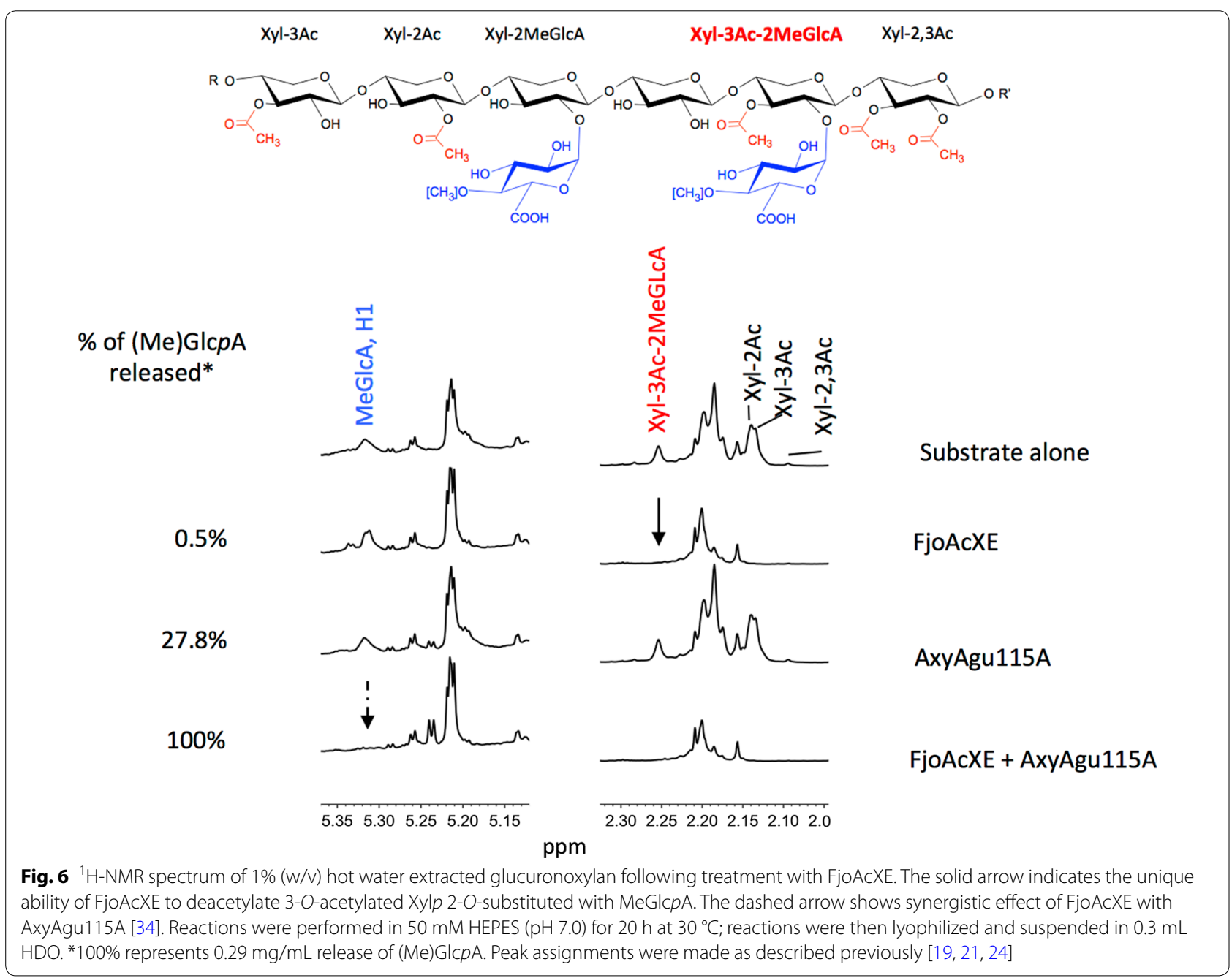




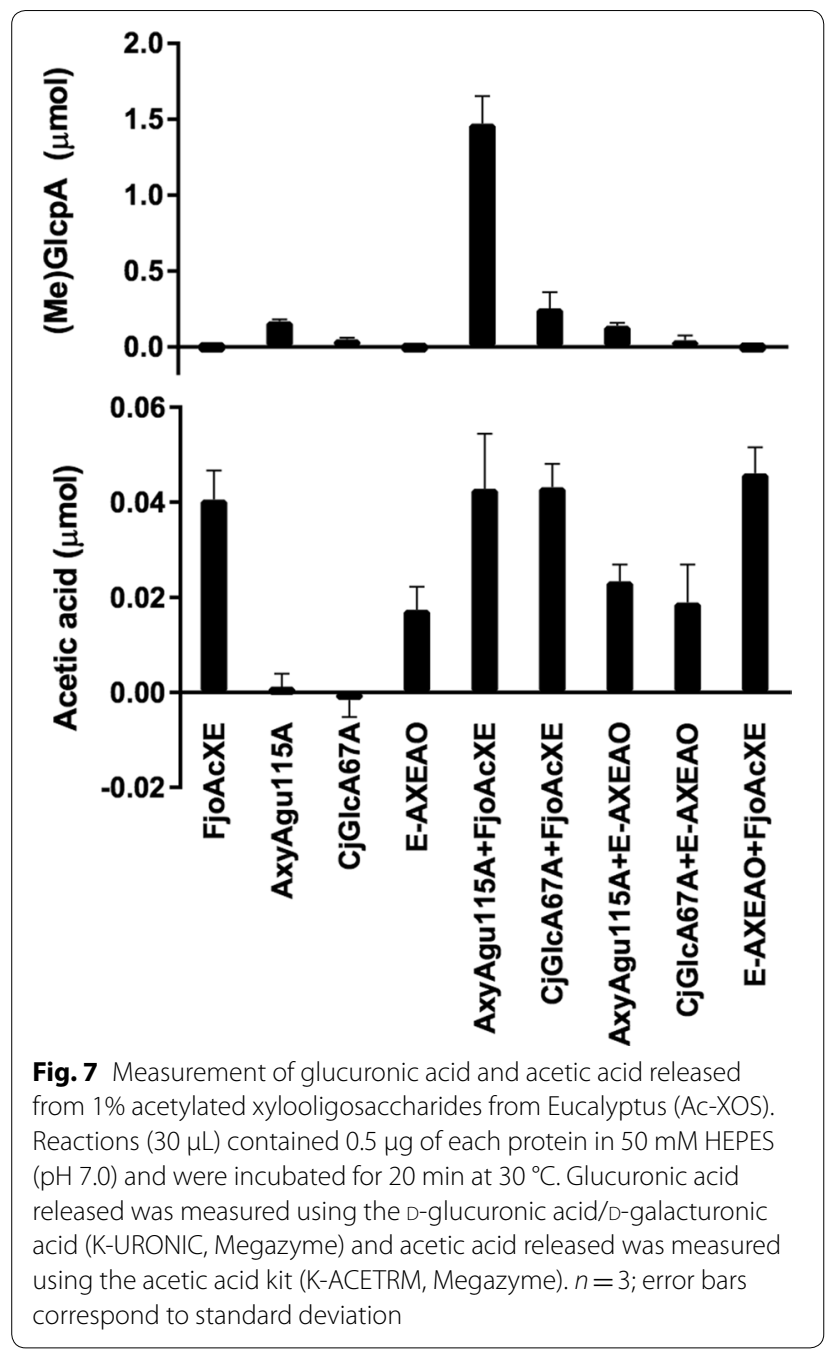

has low susceptibility to steric hindrance. Moreover, activity of FjoAcXE on acetylated xylooligosaccharides supports its designation as an acetyl xylan esterase.

To then evaluate the regioselectivity of FjoAcXE action, acetyl group release from specific positions within oligosaccharides of acetylated glucuronoxylan was monitored by ${ }^{1} \mathrm{H}$-NMR. Remarkably, in addition to targeting 2-O-acetyl-Xyl $p, 3-O$-acetyl-Xyl $p$, and 2,3-O-acetyl Xyl $p$ positions, FjoAcXE fully released the acetyl group from (2-O-MeGlcpA)3-O-acetyl-Xyl $p$ structures (Fig. 6). Such complete activity towards (2-O-MeGlc $p$ A)3-O-acetyl$\mathrm{Xyl} p$ structures has not been previously reported, and shows that FjoAcXE was not impacted by the steric hindrance presented by MeGlcpA that has plagued AcXE enzymes characterized to date.

Action of FjoAcXE towards (2-O-MeGlcpA)3-O-acetyl$\mathrm{Xyl} p$ structures was further explored through synergistic action of FjoAcXE with AxyAgu115A and CjGlcA67A. The commercial CE6, E-AXEAO, which was previously shown to deacetylate glucuronoxylan at all positions except (2-O-MeGlc $p \mathrm{~A}) 3-O$-acetyl-Xyl $p$ structures, was used for comparison [22]. MeGlcpA release by AxyAgu115A from oligosaccharides of acetylated glucuronoxylan (Ac-XOS) increased nearly nine times when in the presence of FjoAcXE (Fig. 7). Similarly, MeGlcpA release from Ac-XOS by CjGlcA67A increased nearly five times in the presence of FjoAcXE. The higher impact of FjoAcXE activity on MeGlcpA release by AxyAgu115A compared to CjGlcA67A is consistent with the ability of FjoAcXE to target both internal and terminal (2-O-MeGlc $p \mathrm{~A}) 3-O$-acetyl-Xyl $p$ structures. By contrast, addition of E-AXEAO did not impact AxyAgu115A or CjGlcA67A activity (Fig. 7), consistent with the lack of E-AXEAO activity towards (2-O-MeGlcpA)3-O-acetylXyl $p$ structures.

\section{MALDI-TOF analysis of FjoAcXE towards xylooligomers from corn fiber}

Given the efficient release of typically recalcitrant (2-O-MeGlcpA)3-O-acetyl-Xyl $p$ structures, FjoAcXE activity was also tested using complex oligosaccharides recovered from feruloyated arabinoxylans, which were previously recovered and described in detail [8]. Based on the earlier, detailed characterization of the corresponding feruloyated xylooligomers by both NMR and ESI-MS, the exact structures transformed by FjoAcXE could be identified herein by MALDI-TOF [8]. In particular, a decrease in acetylated xylo-arabino-oligomers corresponding to peak $(\mathrm{m} / \mathrm{z})$ values 905 and 1037 was identified after treatment with FjoAcXE (Fig. 8). These results indicate that FjoAcXE is also able to target 2-O-acetyl-Xyl $p$ structures where the same Xyl $p$ is substituted at the $O-3$ position by arabinosyl residues; however, 


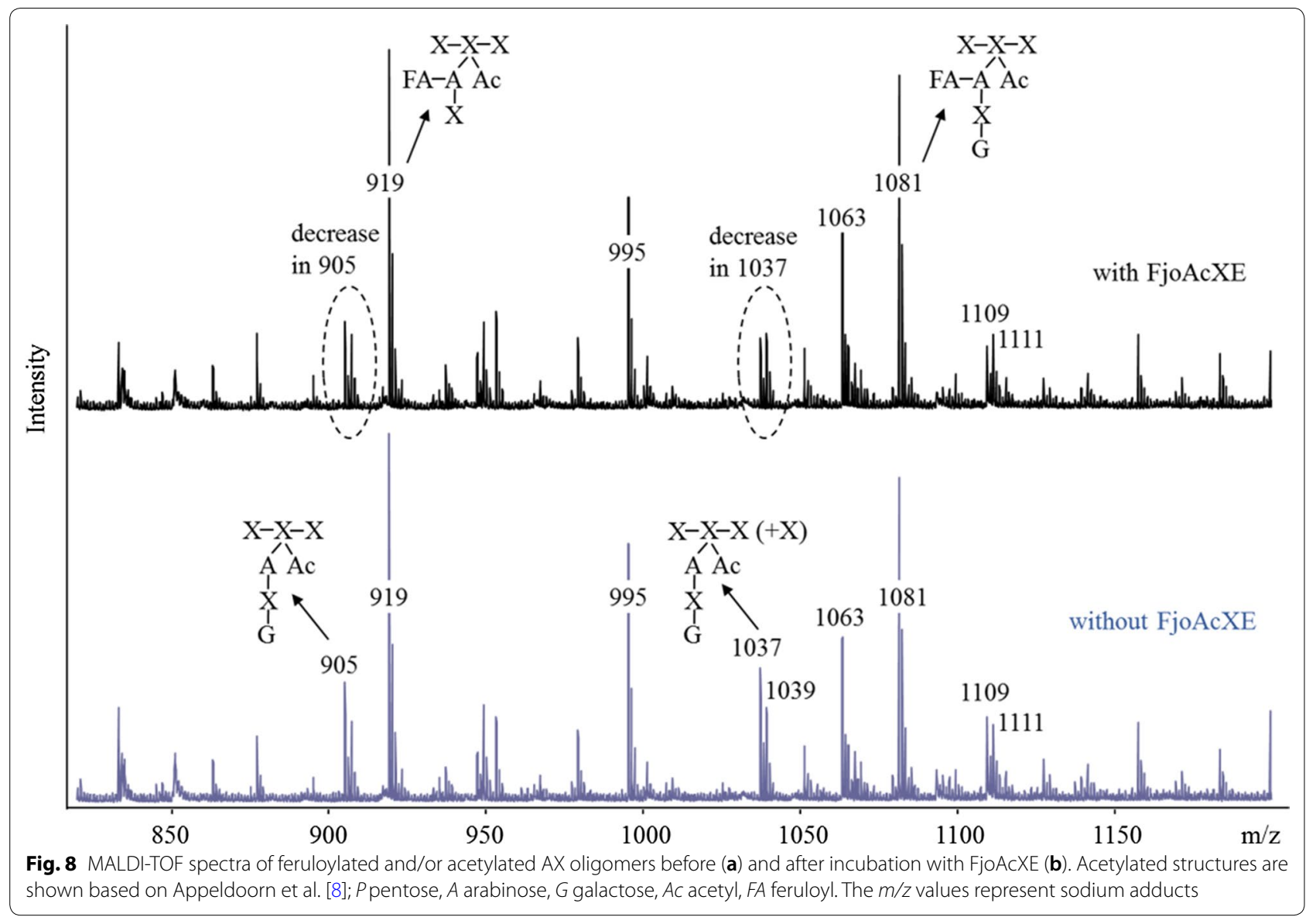

release of the acetyl group that neighbors the feruloylated side group was not detected [8] (Fig. 8).

\section{Conclusions}

An unclassified protein from a polysaccharide utilization locus predicted to promote xylan deconstruction was biochemically characterized and shown to harbor novel carbohydrate esterase activity, which was missing from the arsenal of enzymes required for GX conversion. In addition to promoting $\alpha$-glucuronidase activity through release of acetyl groups from internal (2-O-MeGlcpA)3$O$-acetyl-Xyl $p$ structures, FjoAcXE also targeted
(3-O-Araf)2-O-acetyl-Xyl $p$ of feruloylated xylooligomers. The novel ability of FjoAcXE to release acetyl groups of Xyl $p$ backbone residues that are further substituted by either MeGlc $p$ A or a neutral oligomeric side chain could be explained by the loop regions predicted to surround the catalytic site of the enzyme and to play a role in substrate preference. On-going efforts to solve the FjoAcXE structure, and to characterize predicted homologs of FjoAcXE, will help confirm these predictions and likely uncover additional protein features that determine the broad substrate range of this new carbohydrate esterase activity. 


\section{Additional files}

Additional file 1: Fig. S1. Structural model of FjoAcXE. Top view shows a cartoon only view, button shows CE domain as solvent-exposed surface representation. The CE domain is shown in darker shade, CBM-like domain in lighter shade. The catalytic triad and SGNH motif residues are shown in red, with expected hydrogen bonds between catalytic triad S196D371-H374 shown as dashes. Three loops impinging on the active site cleft are coloured black and labeled. The model was built with Modeller for the CE domain and Phyre2 for the CBM.

Additional file 2: Fig. S2. Affinity gel electrophoresis. $5 \mu \mathrm{g}$ of FjoAcXE was run for $2 \mathrm{~h}$ at $90 \mathrm{~V}$ on $7.5 \%(\mathrm{w} / \mathrm{v})$ native polyacrylamide gel $(25 \mathrm{mM}$ Tris, $250 \mathrm{mM}$ glycine buffer ( $\mathrm{pH} 8.3$ ) containing $0.01 \%$ of each substrate; gels were then stained with Coomassie Blue G. Bovine serum albumin (BSA) was used as a reference. $M G X=4-O-($ methyl)-glucuronoxylan; OSX = oat spelt xylan; $C M C=$ carboxymethylcellulose, $\mathrm{WAX}=$ wheat arabinoxylan.

Additional file 3: Fig. S3. Purified FjoAcXE is approximately 45.2 kDA.

Additional file 4: Fig. S4. FjoAcXE activity screen against $0.5 \%(\mathrm{w} / \mathrm{V})$ of selected polysaccharides. Reactions $(50 \mu \mathrm{L}$ ) contained $5 \mu \mathrm{g}$ of FjoAcXE, $50 \mathrm{mM}$ HEPES ( $\mathrm{pH}$ 8.0), and $0.5 \% \mathrm{w} / \mathrm{v}$ of each substrate, and were incubated for $16 \mathrm{~h}$ at $30^{\circ} \mathrm{C}$. Reducing sugars were measured using $1 \%$ final PAHBAH reagent [58]. BEX = beechwood $x y$ lan (Sigma, $X 4252) ; O S X=0 a t$ spelt xylan (Sigma, X0627); CMC = carboxymethylcellulose (Megazyme, P-CMC4 M); $\beta$-glucan (low viscosity; from barley; Megazyme, P-BGBL); starch (from corn; Sigma-Aldrich, S4126); pectin (from apple; Sigma, 76282); WAX = wheat arabinoxylan (high viscosity; Megazyme, P-WAXYH); arabinan (from sugarbeet; Megazyme, P-ARAB); glucomannan (low viscosity; from konjac; Megazyme, P-GLCML); galactomannan (from guar, GD28; Megazyme, enzyme modified); xyloglucan (amyloid, from tamarind seed; Megazyme, P-XYGLN); arabinogalactan (acacia gum, Sigma, G9752).

Additional file 5: Fig. S5. FjoAcXE activity screen against selected $p N P$ substrates. Reactions $(200 \mu \mathrm{L})$ contained $5 \mu \mathrm{g}$ of FjOAcXE, $50 \mathrm{mM}$ HEPES ( $\mathrm{pH} 8.0)$, and $2 \mathrm{mM}$ of each substrate. Absorbance was measured after $2 \mathrm{~h}$ at $30^{\circ} \mathrm{C}$.

Additional file 6: Fig. S6. Screen of FjoAcXEA activity towards selected $p N P$ alkyl esters showing activity on short chain $(<C 4)$ substrates consistent with esterase rather than lipase activity. Reactions $(200 \mu \mathrm{L})$ contained $0.5 \mu \mathrm{g}$ of FjoAcXE, $50 \mathrm{mM}$ HEPES ( $\mathrm{pH}$ 8.0), and $2 \mathrm{mM}$ of each substrate. Absorbance at $410 \mathrm{~nm}$ was measured after $2 \mathrm{~h}$ at $30^{\circ} \mathrm{C}$. pNP acetate $\left(\mathrm{C}_{2}\right)$, $p N P$ butyrate $\left(C_{4}\right), p N P$ hexanoate $\left(C_{6}\right), p N P$ octanoate $\left(C_{8}\right), p N P$ decanoate $\left(C_{10}\right), p N P$ dodecanoate $\left(C_{12}\right), p N P$ myristate $\left(C_{14}\right)$, and $p N P$ palmitate $\left(C_{16}\right)$. $n=3$; error bars correspond to standard deviation.

\section{Authors' contributions}

FMR selected, recombinantly expressed, purified, and characterized FjoAcXE, and wrote the manuscript. EJ analyzed FjoAcXE activity on feruloylated xylooligosaccharides by MALDI-TOF/TOF and contributed to data interpretation. PS developed and interpreted the FjoACXE model structure. MT and MAK provided characterized acetylated glucurono-xylooligosaccharides and feruloylated xylo-oligosaccharides, respectively, and assisted with NMR and mass spectrometry analyses. RY and WW contributed to the basic characterization of FjoAcXE characterization, provision of AxyAgu115A, and 1H NMR analyses. ERM conceived and coordinated the study. All authors contributed to the revision of manuscript. All authors read and approved the final manuscript.

\footnotetext{
Author details

${ }^{1}$ Department of Chemical Engineering and Applied Chemistry, University of Toronto, 200 College Street, Toronto, ON M5S 3E5, Canada. ${ }^{2}$ Department of Bioproducts and Biosystems, Aalto University, Kemistintie 1, 00076 Aalto Espoo, Finland. ${ }^{3}$ Department of Food and Environmental Sciences, University of Helsinki, P.O. Box 66, 00014 Helsinki, Finland. ${ }^{4}$ Laboratory of Food Chemistry, Wageningen University, Bornse Weilanden 9, 6708 WG Wageningen, The Netherlands.
}

\section{Acknowledgements}

We thank Professor B. Saville (University of Toronto) for preparing hot water extracted glucuronoxylan from mixed hardwood, which was used to advance the characterization of FjoAcXE.

\section{Competing interests}

The authors declare that they have no competing interests.

\section{Availability of data and materials}

All data generated or analyzed during this study are included in this published article [and its additional files].

\section{Consent for publication}

Not applicable.

\section{Ethics approval and consent to participate}

Not applicable.

\section{Funding}

This work was funded by the Natural Sciences and Engineering Research Council of Canada (NSERC) for the Strategic Network Grant "Industrial Biocatalysis Network,"the European Research Council (ERC) Consolidator Grant to ERM (BHIVE-648925), and an NSERC Canada Graduate Scholarship Masters Award and the Ontario Graduate Scholarship to FMR.

\section{Publisher's Note}

Springer Nature remains neutral with regard to jurisdictional claims in published maps and institutional affiliations.

Received: 27 December 2017 Accepted: 9 March 2018

Published online: 22 March 2018

\section{References}

1. Mishra A, Malhotra AV. Tamarind xyloglucan: a polysaccharide with versatile application potential. J Mater Chem. 2009;19:8528.

2. Hansen NML, Plackett D. Sustainable films and coatings from hemicelIuloses: a review. Biomacromolecules. 2008;9:1493-505.

3. Ebringerová A. Structural diversity and application potential of hemicelluloses. Macromol Symp. 2006;232:1-12.

4. Teleman A, Tenkanen M, Jacobs A, Dahlman O. Characterization of O-acetyl-(4-O-methylglucurono)xylan isolated from birch and beech. Carbohydr Res. 2002;337:373-7.

5. Kabel MA, De Waard P, Schols HA, Voragen AGJ. Location of O-acetyl substituents in xylo-oligosaccharides obtained from hydrothermally treated Eucalyptus wood. Carbohydr Res. 2003;338:69-77.

6. Teleman A, Lundqvist J, Tjerneld F, Stalbrand H, Dahlman O. Characterization of acetylated 4-O-methylglucuronoxylan isolated from aspen employing ${ }^{1} \mathrm{H}$ and ${ }^{13} \mathrm{C}$ NMR spectroscopy. Carbohydr Res. 2000;329:807-15.

7. Biely P, Singh S, Puchart V. Towards enzymatic breakdown of complex plant xylan structures: state of the art. Biotechnol Adv. 2016;34:1260-74.

8. Appeldoorn MM, De Waard P, Kabel MA, Gruppen H, Schols HA. Enzyme resistant feruloylated xylooligomer analogues from thermochemically treated corn fiber contain large side chains, ethyl glycosides and novel sites of acetylation. Carbohydr Res. 2013;381:33-42.

9. Gírio FM, Fonseca C, Carvalheiro F, Duarte LC, Marques S, Bogel-Łukasik R. Hemicelluloses for fuel ethanol: a review. Bioresour Technol. 2010;101:4775-800.

10. Ebringerova A, Hromadkova Z, Heinze T. Hemicellulose. Adv Polym Sci. 2005;186:1-67.

11. Appeldoorn MM, Kabel MA, Van Eylen D, Gruppen H, Schols HA. Characterization of oligomeric xylan structures from corn fiber resistant to pretreatment and simultaneous saccharification and fermentation. J Agric Food Chem. 2010;58:11294-301.

12. Littunen K, Mai-Gisondi G, Seppälä J, Master ER. Enzymatically debranched xylans in graft copolymerization. Biomacromol. 2017;18:1634-41.

13. Lombard V, Golaconda Ramulu H, Drula E, Coutinho PM, Henrissat B. The carbohydrate-active enzymes database (CAZy) in 2013. Nucleic Acids Res. 2014;42:490-5. 
14. Nakamura AM, Nascimento AS, Polikarpov I. Structural diversity of carbohydrate esterases. Biotechnol Res Innov. 2017;1:35-51.

15. Alalouf O, Balazs Y, Volkinshtein M, Grimpel Y, Shoham G, Shoham Y. A new family of carbohydrate esterases is represented by a GDSL hydrolase/acetylxylan esterase from Geobacillus stearothermophilus. J Biol Chem. 2011;286:41993-2001.

16. Biely P, Puls J, Schneider H. Acetyl xylan esterases in fungal cellulolytic systems. FEBS Lett. 1985;186:80-4

17. Biely P. Microbial carbohydrate esterases deacetylating plant polysaccharides. Biotechnol Adv. 2012;30:1575-88.

18. Adesioye FA, Makhalanyane TP, Biely P, Cowan DA. Phylogeny, classification and metagenomic bioprospecting of microbial acetyl xylan esterases. Enzyme Microb Technol. 2016;93:79-91.

19. Neumüller KG, de Souza AC, van Rijn JH, Streekstra H, Gruppen H, Schols HA. Positional preferences of acetyl esterases from different CE families towards acetylated 4-O-methyl glucuronic acid-substituted xylo-oligosaccharides. Biotechnol Biofuels. 2015;8:7.

20. Puchart V, Berrin JG, Haon M, Biely P. A unique CE16 acetyl esterase from Podospora anserina active on polymeric xylan. Appl Microbiol Biotechnol. 2015:99:10515-26.

21. Puchart V, Agger JW, Berrin JG, Várnai A, Westereng B, Biely P. Comparison of fungal carbohydrate esterases of family CE16 on artificial and natural substrates. J Biotechnol. 2016;233:228-36.

22. Mai-Gisondi G, Maaheimo H, Chong SL, Hinz S, Tenkanen M, Master E. Functional comparison of versatile carbohydrate esterases from families CE1, CE6 and CE16 on acetyl-4-O-methylglucuronoxylan and acetyl-galactoglucomannan. Biochim Biophys Acta Gen Subj. 2017;1861:2398-405.

23. Puchart V, Biely P. Redistribution of acetyl groups on the non-reducing end xylopyranosyl residues and their removal by xylan deacetylases. Appl Microbiol Biotechnol. 2014;99:3865-73.

24. Uhliariková I, Vršanská M, McCleary BV, Biely P. Positional specifity of acetylxylan esterases on natural polysaccharide: an NMR study. Biochim Biophys Acta Gen Subj. 2013;1830:3365-72.

25. Grondin JM, Tamura K, Déjean G, Abbott DW, Brumer H. Polysaccharide utilization loci: fuelling microbial communities. J Bacteriol. 2017;199:e00860-16.

26. Terrapon N, Lombard V, Gilbert HJ, Henrissat B. Automatic prediction of polysaccharide utilization loci in Bacteroidetes species. Bioinformatics. 2014;31:647-55.

27. Ndeh D, Rogowski A, Cartmell A, Luis AS, Baslé A, Gray J, et al. Complex pectin metabolism by gut bacteria reveals novel catalytic functions. Nature. 2017;544:65-70.

28. Wang K, Pereira GV, Cavalcante JJV, Zhang M, Mackie R, Cann I. Bacteroides intestinalis DSM 17393, a member of the human colonic microbiome, upregulates multiple endoxylanases during growth on xylan. Sci Rep. 2016;6:34360

29. Bagenholm V, Reddy SK, Bouraoui H, Morrill J, Kulcinskaja E, Bahr CM, et al. Galactomannan catabolism conferred by a polysaccharide utilization locus of Bacteroides ovatus: enzyme synergy and crystal structure of a B-mannanase. J Biol Chem. 2017:292:229-43.

30. Larsbrink J, Zhu Y, Kharade SS, Kwiatkowski KJ, Eijsink VGH, Koropatkin NM, et al. A polysaccharide utilization locus from Flavobacterium johnsoniae enables conversion of recalcitrant chitin. Biotechnol Biofuels. 2016:9:260.

31. Temple MJ, Cuskin F, Baslé A, Hickey N, Speciale G, Williams SJ, et al. A Bacteroidetes locus dedicated to fungal 1,6- $\beta$-glucan degradation: unique substrate conformation drives specificity of the key endo-1,6- $\beta$ glucanase. J Biol Chem. 2017;292:10639-50.

32. Gullón P, González-Muñoz MJ, Domínguez H, Parajó JC. Membrane processing of liquors from Eucalyptus globulus autohydrolysis. J Food Eng. 2008:87:257-65

33. Yan R. Characterization of novel GH115 alpha-glucuronidases for enzymatic tailoring of xylans. Toronto: Univeristy of Toronto; 2017.

34. Yan R, Vuong TV, Wang W, Master ER. Action of a GH115 a-glucuronidase from Amphibacillus xylanus at alkaline condition promotes release of 4-O-methylglucopyranosyluronic acid from glucuronoxylan and arabinoglucuronoxylan. Enzyme Microb Technol. 2017;104:22-8.

35. Petersen TN, Brunak S, von Heijne G, Nielsen H. SignalP 4.0: discriminating signal peptides from transmembrane regions. Nat Methods. 2011:8:785-6.
36. Kelly LA, Mezulis S, Yates C, Wass M, Sternberg M. The Phyre2 web portal for protein modelling, prediction, and analysis. Nat Protoc. 2015:10:845-58.

37. Webb B, Sali A. Protein structure prediction. Curr Protoc Bioinform. 2014;1137:1-15.

38. Mølgaard A, Larsen S. A branched N-linked glycan at atomic resolution in the $1.12 \AA$ structure of rhamnogalacturonan acetylesterase. Acta Crystallogr Sect Biol Crystallogr. 2002;58:111-9.

39. Bradford MM. A rapid and sensitive method for the quantitation of microgram quantities of protein utilizing the principle of protein-dye binding. Anal Biochem. 1976;72:248-54.

40. Shevchenko A, Tomas H, Havlis J, Olsen JV, Mann M. In-gel digestion for mass spectrometric characterization of proteins and proteomes. Nat Protoc. 2006:1:2856-60

41. Mai-Gisondi G, Master ER. Colorimetric detection of acetyl xylan esterase activities. Methods Mol Biol. 2017:1588:45-57.

42. Wang W, Mazurkewich S, Kimber MS, Seah SYK. Structural and kinetic characterization of 4-hydroxy-4-methyl-2- oxoglutarate/4-carboxy-4-hydroxy-2-oxoadipate aldolase, a protocatechuate degradation enzyme evolutionarily convergent with the Hpal and DmpG pyruvate aldolases. J Biol Chem. 2010;285:36608-15.

43. Montanier C, Money VA, Pires VMR, Flint JE, Pinheiro BA, Goyal A, et al. The active site of a carbohydrate esterase displays divergent catalytic and noncatalytic binding functions. PLoS Biol. 2009;7:0684-97.

44. Ašler IL, Ivić N, Kovačić F, Schell S, Knorr J, Krauss U, et al. Probing enzyme promiscuity of SGNH hydrolases. ChemBioChem. 2010;11:2158-67.

45. Liu AMF, Somers NA, Kazlauskas RJ, Brush TS, Zocher F, Enzelberger MM, et al. Mapping the substrate selectivity of new hydrolases using colorimetric screening: lipases from Bacillus thermocatenulatus and Ophiostoma piliferum, esterases from Pseudomonas fluorescens and Streptomyces diastatochromogenes. Tetrahedron Asymmetry. 2001;12:545-56.

46. Bakshy K, Gummadi SN, Manoj N. Biochemical characterization of Alr1529, a novel SGNH hydrolase variant from Anabaena sp. PCC 7120. Biochim Biophys Acta Proteins Proteomics. 2009;1794:324-34.

47. Hwang H, Kim S, Yoon S, Ryu Y, Lee SY, Kim TD. Characterization of a novel oligomeric SGNH-arylesterase from Sinorhizobium meliloti 1021. Int J Biol Macromol. 2010;46:145-52.

48. Kim S, Bae SY, Kim SJ, Ngo TD, Kim KK, Kim TD. Characterization, amyloid formation, and immobilization of a novel SGNH hydrolase from Listeria innocua 11262. Int J Biol Macromol. 2012;50:103-11.

49. Bae SY, Ryu BH, Jang E, Kim S, Kim TD. Characterization and immobilization of a novel SGNH hydrolase (Est24) from Sinorhizobium meliloti. Appl Microbiol Biotechnol. 2013;97:1637-47.

50. Yu T, Ding J, Zheng Q, Han N, Yu J, Yang Y, et al. Identification and characterization of a new alkaline SGNH hydrolase from a thermophilic bacterium Bacillus sp. K91. J Microbiol Biotechnol. 2016;26:730-8.

51. Panda T, Gowrishankar BS. Production and applications of esterases. Appl Microbiol Biotechnol. 2005;67:160-9.

52. Mølgaard A, Kauppinen S, Larsen S. Rhamnogalacturonan acetylesterase elucidates the structure and function of a new family of hydrolases. Structure. 2000:8:373-83.

53. Lo Y-C, Lin S-C, Shaw J-F, Liaw Y-C. Crystal structure of Escherichia coli thioesterase I/protease I//ysophospholipase L1: consensus sequence blocks constitute the catalytic center of SGNH-hydrolases through a conserved hydrogen bond network. J Mol Biol. 2003;330:539-51.

54. Lee YL, Chen JC, Shaw JF. The thioesterase I of Escherichia coli has arylesterase activity and shows stereospecificity for protease substrates. Biochem Biophys Res Commun. 1997;231:452-6.

55. Kim K, Ryu BH, Kim SS, An DR, Ngo TD, Pandian R, et al. Structural and biochemical characterization of a carbohydrate acetylesterase from Sinorhizobium meliloti 1021. FEBS Lett. 2015;589:117-22.

56. Till M, Goldstone DC, Attwood GT, Moon CD, Kelly WJ, Arcus VL. Structure and function of an acetyl xylan esterase (Est2A) from the rumen bacterium Butyrivibrio proteoclasticus. Proteins Struct Funct Bioinform. 2013:81:911-7.

57. Katoh K, Misawa K, Kuma K, Miyata T. MAFFT: a novel method for rapid multiple sequence alignment based on fast Fourier transform. Nucleic Acids Res. 2002;30:3059-66.

58. Lever M. A new reaction for calorimetric determination of carbohydrates. Anal Biochem. 1972:47:273-9. 